\title{
Design and Analysis of Micro-Cantilever Based Biosensor for Swine Flu Detection
}

\author{
M.G.G. Jithendra Prasad, Syed Shameem
}

Department of Electronics and communication Engineering, K L University, A.P, India

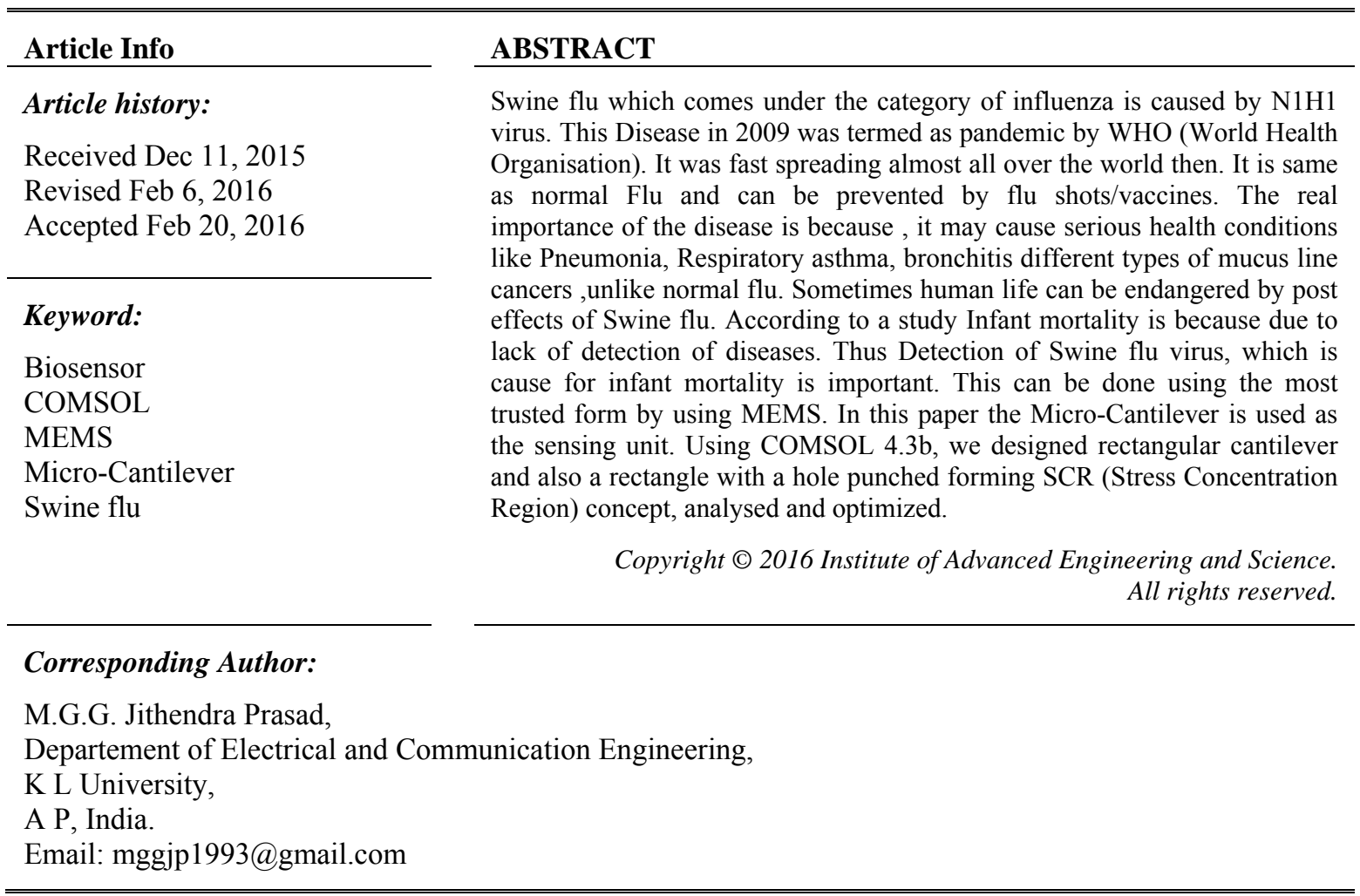

\section{INTRODUCTION}

The microelectronic mechanical cantilevers [1],[2] is one of the most promising and trustable biosensors. Cantilever provides an outstanding platform for extremely sensitive process like chemical sensing, [3] biological sensing [4] occasions. Micro cantilevers are popularly known for their sensitivity \& selectivity. It also provides us compatibility features like flexibility of a chip circuit, easily deployable into an IC. It is popular because of ease in fabrication like other conventional devices there is no need to separate external modules for detection or sensing, cantilevers when fabricated using mass production it yields a numerous amount of economical features like low cost, flexibility in production \& versatility. Both the selectivity and the sensitivity play a key role in accounting the quality of the sensor. Micro cantilever may be used to sense different analytes Glucose [5], fungal detections [6] etc., like Using the Micro-Cantilever we can achieve both of the above characteristics. Antigen-Antibody pairing is a natural gift of the science used in attaining the Selectivity [7],[8]. The detection principle of Micro cantilever may differ depending on user constraints like mass differentiations [9], frequency changes [10] etc., For improving the sensitivity of the sensor we can use different approaches like increasing the length, decreasing the thickness, changing the materials, according to Stone's Law. In this paper, we are using a concept termed as SCR [11] (stress concentration regions) to increase the sensitivity. We can punch any required shape so as to change the Sensitivity [12], Also using this sensor so as to contribute it for an Detection of the Virus which causes the Swine flu is our major portion. Swine flu is common flu, but if neglected it causes serious health crisis for human [13]. In worst case scenario it may also lead to deadly diseases like Pneumonia, bronchits, lung 
cancer, throat cancer etc. Thus, if we differentiate the swine flu from other normal flu then it may save a large number of human lives.

\section{DESIGN CONSTRAINTS}

Physical representation of a cantilever is nothing but a shape anchored at any one of its end. For example, rectangular, triangular, trapezoidal, $\mathrm{v}$-shaped, $\mathrm{t}$-shaped, $\pi$ shaped etc., beams are fixed to a end (face). The design parameters of cantilever is based on three formulae [14] (i)Stone's formula (ii)Spring constant formula (iii)Hooke's law. The above equation provides us a key source to understand the behavior of the MEMS cantilevers. Briefing the above equations here we have,

(i) Stone's formula:

It is used to calculate and analyse the deflection of the micro cantilever. It is given by the equation

$$
\delta=\frac{3 \sigma(1-\vartheta)}{e}(E \xi t)^{2}
$$

Here $\vartheta$ is Poisson's ratio; $\in$ is Young's modulus; $\mathrm{L}$ is Beam length; $\mathrm{t}$ is Cantilever thickness; $\sigma$ is applied stress; $\delta$ is Deflection.

The deflection of the cantilever depends on dimensions of the structure and the materials used for construction of the structure. It also decides on characteristic features like the stiffness of the material used in construction, amount of target molecules, so as to observe and analyze the significant amount of the deflection. Transduction principles are used in order to detect deflections which are small. The below listed are few of methods. (i)Optical readout (ii)Capacitive readout (iii)Hard contact/tunnelling (iv)Autonomous devices [15].

\section{(ii) Spring Constant (K)}

The second formula relates to spring constant ' $\mathrm{K}$ ', which plays a major role in analysing the

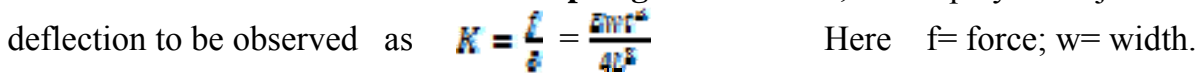

\section{(iii) Hooke's law:}

Hooke's law gives us a relation between the force applied to the displacement. Thus the amount of the extra mass exerts a force on the cantilever beam thus the displacement of the beam increases considerably. Hooke's law can be mathematically represented as $\mathrm{F}=-\mathrm{KX}$. The negative symbol is due to representing the restoring force exerted.

\section{PRINCIPLE OF BIOSENSOR USING MICROCANTILEVER}

With its working the guideline of changing over of bio acknowledgment to nano-mechanical movement, the microcantilever based biosensors results in free vitality change [4]. The free vitality change causes the nano mechanical movement on surface of cantilever because of response of target analyte with test covering particle. To recognize a particular analyte, the miniaturized scale cantilever transducer is forged with test covering at first glance taking into account the way of the analyte. The test covering is an artificially touchy layer that gives specificity to analyte acknowledgment. The main concept is absolutely in view of the on change causing substance/organic boosts up into mechanical reaction. Cantilever covering is the thing that analyte atom diffuse into, in the perspective of presenting it to analyte vapor. The covering starts to oscillate with the climb of mass. A move of interfacial anxiety in the middle of covering and cantilever happen bringing about a oscillations (Deflection) of cantilevers. With metal coatings alongside self-collected monolayers or polymer, Substance responses are transduced by affectability of cantilevers into a mechanical reaction. Reducing the foundation signals which is allowed by reference cantilever sensor (differential estimation) is imperative.

Cantilever sensor has diverse coatings for distinctive analyte recognition. In Figure 1 the components of bio sensor which is shown. Small scale cantilever here is utilized for bio identification. At the point when a particular organic response happens on a surface of a miniaturized scale cantilever, the subsequent change in surface anxiety avoids the cantilever shaft. A little range is test covering at the free end of the small scale cantilever to catch relating target antibodies. It is the point at which the gadget is presented to an objective hostile to bodied environment, that the covered counter acting agent (on free end of small scale cantilever) will catch them, recommending in a reverberation recurrence movement or static bowing which is identified by distinctive transduction instrument. The mass determination got with cantilevers in air 
is in the Pico and the femto gram range. Mass change on the cantilever can precisely be controlled by running the instrument in static mode and element mode. Polymer layer is utilized to coat on the top surface of cantilever. Unpredictable vaporous analytes are distinguished by dissemination of the atoms into the polymer layer, bringing about free vitality era and there with Deflecting of the cantilevers. Furthermore, in the end, a normal for analyte is acquired from the Deflecting example of all cantilevers.

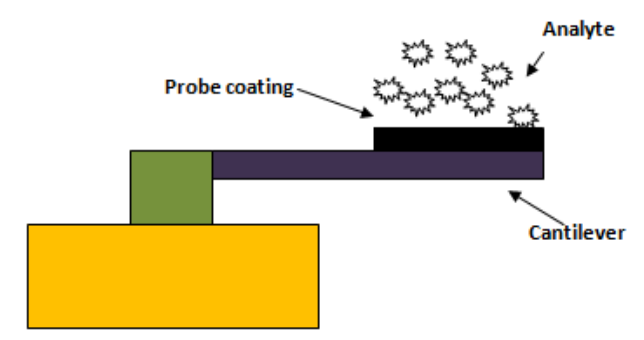

Figure 1. The schematic of Cantilever in bio sensing

\section{BIOSENSING MECHANISM USING MICROCANTILEVER}

It is a fact that most life threatening diseases are detected only after the secondary symptoms sets in, making it tough for the treatment. The rate at which a person gets affected by a disease is high, considering the growing pollution and a changed lifestyle. The scenario reiterates on the need to monitor human body on a regular basis, to stay away from the diseases. The natural resources which used to be very pure are now full of pollutants, courtesy the global warming. All the situations make it important for a developed technology in bio medical field where accurate detection of the disease at the earliest is important. Microcantilever based biosensor which is utilized to sense the vicinity of a sure molecule is the correct choice. Microcantilever analyte ought to be covered with an artificially touchy material to accommodate a high level of specificity in recognizing certain particles or analytes inside of an example. Bio particles can be utilized as a part of covering the cantilever, which recognizes particular analytes inside of a little blood test fastidiously. The characterized sicknesses, from this time forward can be treated with the choice of suitable specification. Microcantilevers are used as biosensors in detecting a disease, which gives scope for miniaturization and parallelization. A lot many samples including antibodies, proteins, antigens [4] and DNA can be analyzed through biosensors. It is already in use in glucose monitors, $\mathrm{pH}$ sensors, protein binding, DNA detection and gene expression profiling Life threatening virus that cause diseases like HIV, tuberculosis can be detected in the primary stage of it. Surface reaction takes place when the analytes are confined to the surface of the probe coating. Generally with a typical length of 10 to $500 \mu \mathrm{m}$ long and width of $100 \mu \mathrm{m}$ wide with $2 \mu \mathrm{m}$ thick as shown in Figure 2.

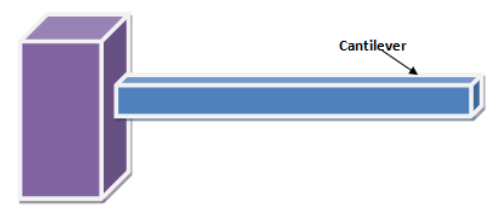

Figure 2. Conventional Rectangular Cantilever with a support block

Microcantilevers could create a transition in medical era. A Schematic side perspective of a microcantilever displaying cantilever length L. The top or base surface or both surfaces are covered with a synthetically responsive material planned particularly for the focused on analyte. A sensor can be utilized by outlining a cantilever biosensor with an alternate test coat [7],[8]. A few substances ought to be at first recognized with the same specimen as in Figure 3. Target material will be distinguished when the cantilever based biosensor is reached with a synthetically touchy material on cantilevers surface. A measure of target material is measured by checking an adjustment in one or a greater amount of the cantilevers mechanical and electrical properties, for example, dislodging, resistance or full recurrence. An adjustment in the mechanical or electrical properties is watched when an analyte ties with the test covering on a cantilevers surface. 


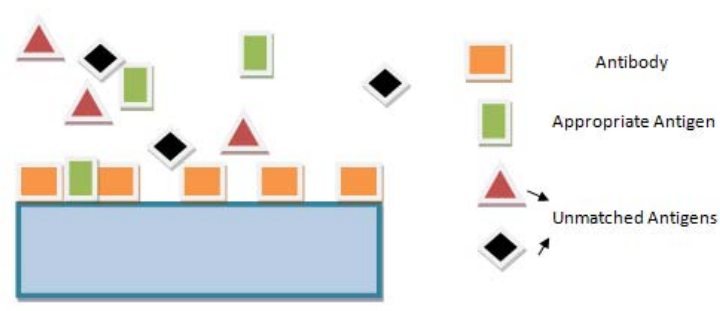

Figure 3. Antibody and Antigen pair Reaction

The transducer changes it's property when more target materials are appended to the cantilevers surface. The subsequent change will be measures. The quantity of analyte or particle ties in the test covering is corresponding to the bowing of the microcantilever. This move is handled by coordinated circuit or flag preparing circuits of MEMS into relative information. The information in the wake of breaking down will be contrasted with reference information to decide the sort of analyte and its properties. The affectability of a microcantilever construct biosensor depends in light of the affectability of the diversion estimation framework. A microcantilever outline ought to have effective transformation from the biomolecular boost into a substantial microcantilever redirection, the estimation affectability guarantees the diversions measured in light of the biomolecular connection and not because of some source. The affectability of the small scale cantilever can be ad libbed by changing the configuration traits, for example, surface push, a sub-atomic association, changing so as to lessen the firmness of the cantilever or the flexible modulus of the cantilever materials.

\section{BREIFING THE ANTIGEN \& ANTIBODY}

(i) SWINE FLU: The morphology [16] of swine influenza virus resembles something as a sphere having a diameter of 75-150nm with pleomorphism as a common feature. The core of virus comprises majorly two things namely RNA genome which is been segmented and existing as eight pieces, along with this RNA independent polymerase which plays a crucial role for transcription of a virus in a favourable condition (inside a host cell). The core is commonly a ribonucleus protein. This nucleocapsule is surrounded by two layer forming envelope. The inner side of the layer is made of protein layer, whereas the outer layer is made of lipid. The inner side envelope is generally coded according to the RNA sequence of the virus, where as the outer side lipid layer will be altered according to the host cell in which replication/culture of the virus is accumulated. Now coming the most important part in virus related to the sensing application is the antigens present on the virus. Majorly classified the antigens on the virus are of two types namely (1) Hemagglutinin (2) Neuraminidase Compared to Neuraminidase, hemagglutinin is very high in number.

In the below virus diagram in Figure 4, we clear can see the morphology of swine flu virus N1H1 having Core, segments of eight in number, hemagglutinin which are represented as triangular spikes and Neuraminidase resembling mushroom shaped. The molecular weight of swine flu is 222KDa. Neuraminidase will try to reduce the viscosity of the nucleus film lining. This defines that Neuraminidase is the one which reacts first in exsanguinous environment. So we concentrate on this antigen present on the virus Morphology.

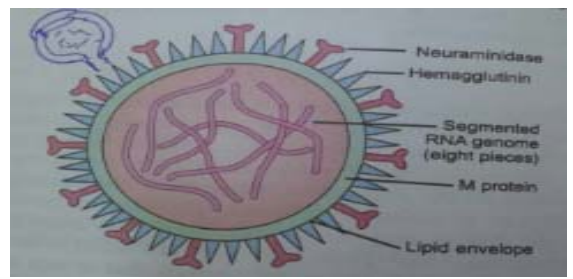

Figure 4. N1H1 Swine flu Virus

(ii) Antibody: For the selectivity we know that the Antigen will be interacting only with the appropriate antibody pair. For Neuraminidase, We have a large number of artificial antibodies using the solutions. But for the Easy understanding we will be considering the Ig A (Immuglobulin A), which is a 
naturally available human secreted antibody. That can bind Swine flu virus using the Neuraminidase Antigen. Ig A is assumed to be the antibody for this because it can be catabolised and have faster response than the Ig $\mathrm{G}$ and its abundant property. Lets us not go much into the isolation of Ig A, We will be just concentrating on the structure of $\operatorname{Ig} \mathrm{A}$, and its part in binding the Antigen particles and the free energy produced by this reaction and the change in deflection. The Antibody Ig A is said to have Dimerism and symmetrical structure that is, it will be having two chains for binding actions, For the Reaction to happen, there must be atleast two Binding sites. Binding sites are nothing but the area at which the proper attachment of the can be taking place. One binding site will be used for the immobilization of the antibody on to the required platform and the second is for the antigen reaction to take place. By the help of Electron microscopy, the most common form observed is y shaped. The heavy Chain also known as $\alpha$ chain is the one which contributes the Molecular mass of the Antibody that is $120 \mathrm{kDa}$. The structure of the Immunoglobulin A will be something resembling like the Figure 5.

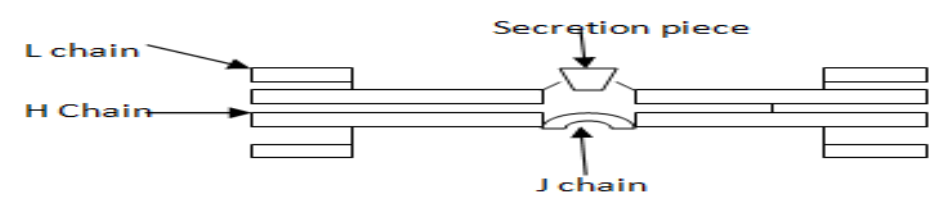

Figure 5. Immuglobulin A

\section{EXPERIMENTAL DESIGN}

A rectangular cantilever beam is assumed with the dimensions $(150,30,1) \mathrm{X} 10^{-6} \mathrm{~m}$. A small rectangular hole $(45,15,1) \times 10^{-6} \mathrm{~m}$ is been punched through the cantilever so as to increase the sensitivity of the Cantilever. This is known as SCR (stress concentration region) concept. The hole is to be punched at the fixed side of the cantilever .this will make the cantilever to deflect than actually meant to be. The Reaction area is constrained as well as the deflection is increased using this principal. Let us now use $\mathrm{SiO} 2, \mathrm{SiC}$, Si3N4, Ploy Si with material properties listed in the Table 1 for optimization.

The surface of the rectangular beam is assumed to have the immobilized antibody probe coating. The initial displacement with a force $3.98 \mathrm{e}-21 \mathrm{~Pa}$ for only coating the probe antibody consisting 20 molecules is noted. Now with an assumption of 20 molecules of antibody is being binded with 10 antigen molecules. The force is calculated for 10 molecules of Antigen and added to existing force with a total of 1.13e-20 Pa and displacement of total displacement with both Antigen and anti body is calculated and noted. By this we can predict that the swine flu virus N1H1 is being attached to the immobilized antibody in this case Ig A. The above calculations are done using COMSOL Multiphysics 4.3b software. For optimization of cantilever we used different materials and calculated the net displacements with initial condition and after reactions on cantilever.

Table 1. Material Properties of used materials

\begin{tabular}{ccccc}
\hline Material Properties & $\mathrm{SiO}_{2}$ & $\mathrm{SiC}$ & $\mathrm{Si}_{3} \mathrm{~N}_{4}$ & Poly Si \\
\hline Density $\left(\mathrm{kg} / \mathrm{m}^{3}\right)$ & 2200 & 3216 & 3100 & 2320 \\
Young's Modulus(Y) $(\mathrm{GPa})$ & 70 & 748 & 250 & 169 \\
Possion's Ratio & 0.17 & 0.45 & 0.23 & 0.22 \\
\hline
\end{tabular}

\section{SIMULATION RESULTS AND DISCUSSIONS}

The results for the above experimental design model Rectangular with Stress Concentration Region(SCR)was observed, For optimization of the design for best results we have used different materials like $\mathrm{SiO} 2, \mathrm{SiC}, \mathrm{Si} 3 \mathrm{~N} 4$, poly $\mathrm{Si}$. $\mathrm{SiO} 2$ was observed to have the largest displacement, which describes for a given assumptions in detection of Swine flu virus, $\mathrm{SiO} 2$ was more sensitive than other elements used. The Simulations are calculated with Antibody alone (initial) and Antibody-Antigen pair combined (After reaction) for $\mathrm{SiO} 2$ material is as shown in Figures 6 and 7 respectively. 


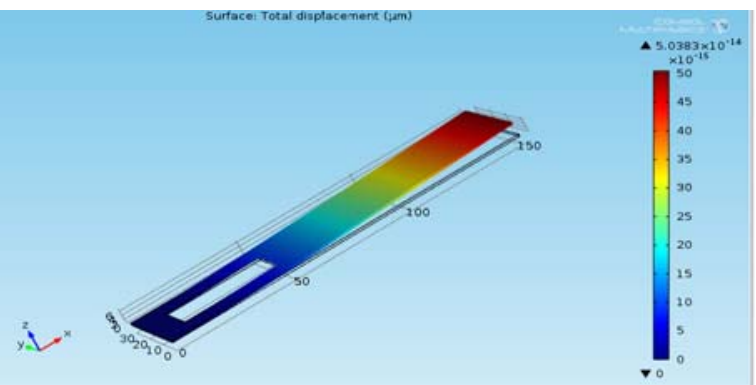

Figure 6. Initial (Antibody alone)

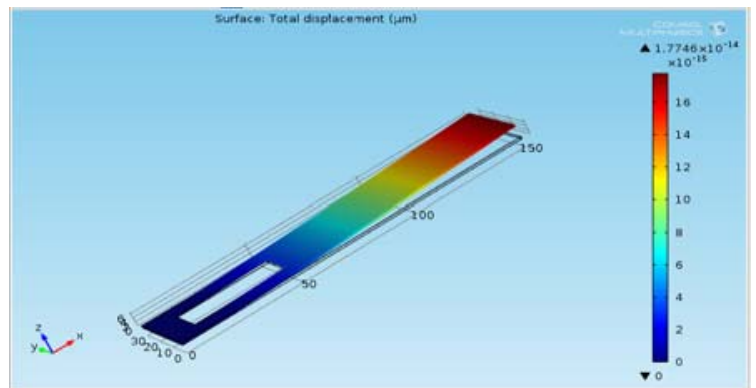

Figure 7. After reaction (both Antibody and Antigen)

The Simulation results of Antibody alone (Initial) and Antibody-Antigen pair (After reaction) for $\mathrm{SiC}$ materials is as shown in Figures 8 and 9 respectively.

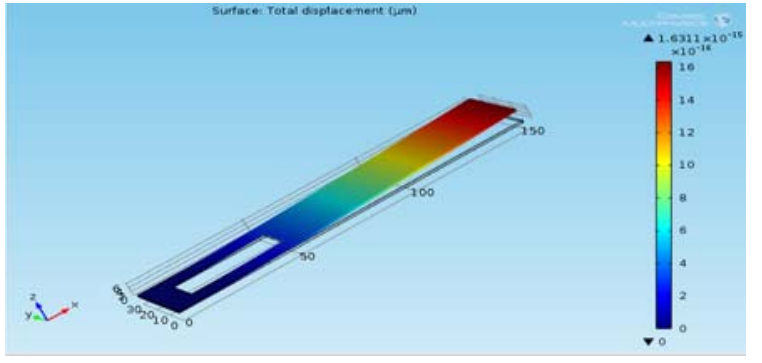

Figure 8. Initial (Antibody alone)

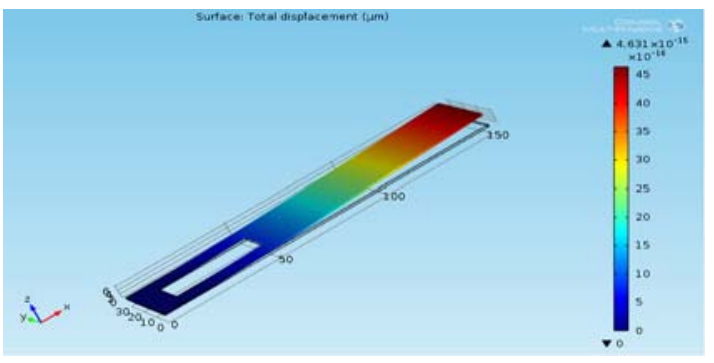

Figure 9. After reaction(both Antibody and Antigen)

The Simulation results of Antibody alone (Initial) and Antibody-Antigen pair (After reaction) for Si3N4 materials is as shown in Figures 10 and 11 respectively.

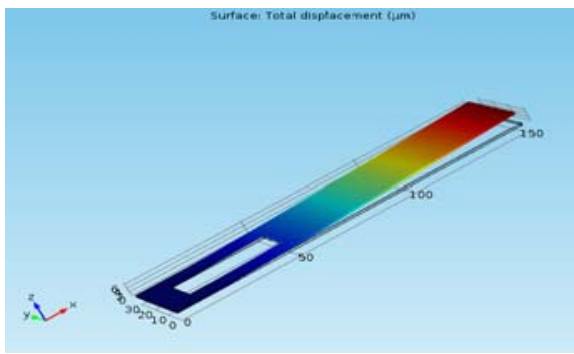

Figure 10. Initial (Antibody alone)
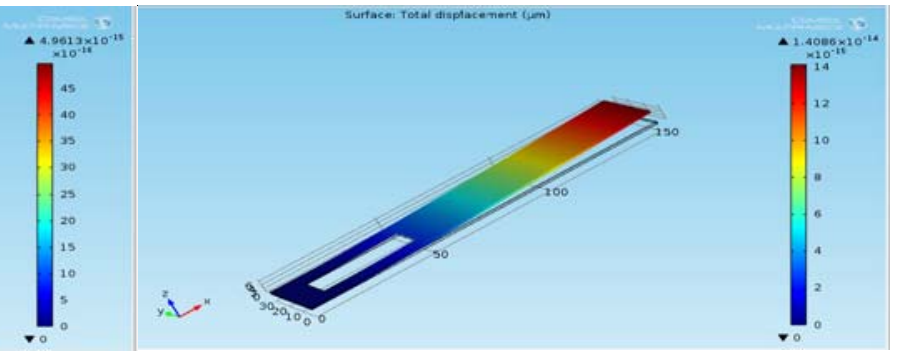

Figure 11. After reaction (both Antibody and Antigen)

The Simulation results of Antibody alone (Initial) and Antibody-Antigen pair (After reaction) for poly Si materials is as shown in Figures 12 and 13 respectively.

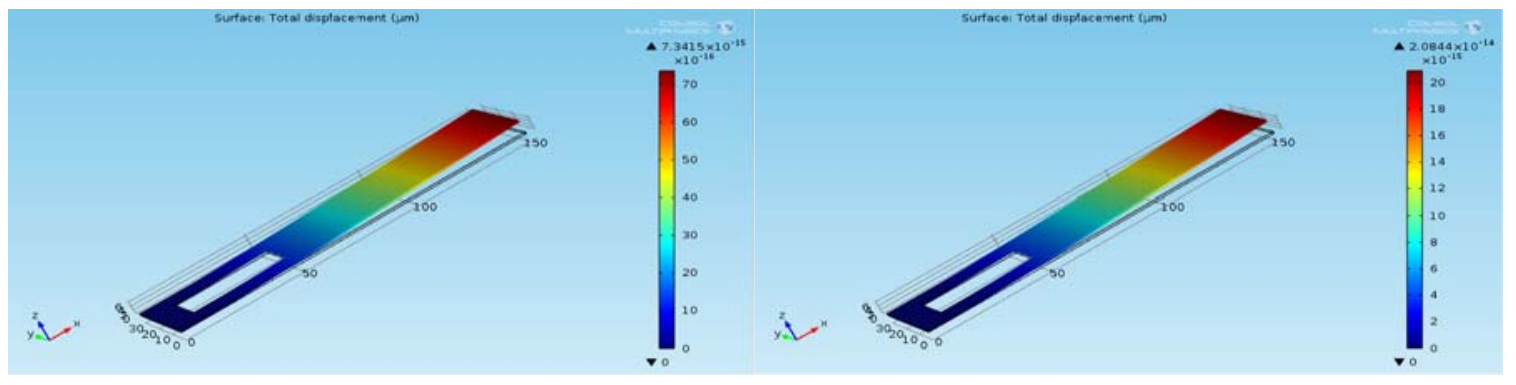

Figure 12. Initial (Antibody alone)

Figure 13. After reaction (both Antibody and Antigen) 
The Table 2 gives us the brief idea of the simulation results with Displacements tabulated for materials used for the Experimental design.

Table 2. Displacements of results

\begin{tabular}{|c|c|c|c|c|}
\hline \multirow{2}{*}{ Displacement } & \multicolumn{3}{|c|}{ Materials } & \multirow[b]{2}{*}{ Poly Si } \\
\hline & $\mathrm{SiC}$ & $\mathrm{SiO}_{2}$ & $\mathrm{Si}_{3} \mathrm{~N}_{4}$ & \\
\hline With Antibody alone $\left(10^{-14} \mu \mathrm{m}\right)$ & 0.163 & 1.7746 & 0.4961 & 0.7341 \\
\hline With both Antibody and Antigen $\left(10^{-14} \mu \mathrm{m}\right)$ & 0.463 & 5.0383 & 1.4086 & 2.0844 \\
\hline
\end{tabular}

We can still increase the sensitivity of the Cantilever by increasing the dimension, suppose the same cantilever is designed using $200 \mu \mathrm{m}$ instead of $150 \mu \mathrm{m}$ then the displacement of the Cantilever is increased to a considerable amount basing Stone's law and results are as shown in Figure 14 and Figure 15 respectively.

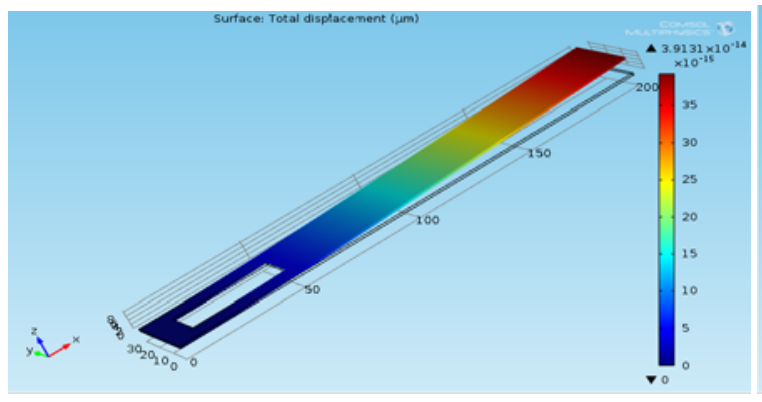

Figure 14. Initial (Antibody alone)

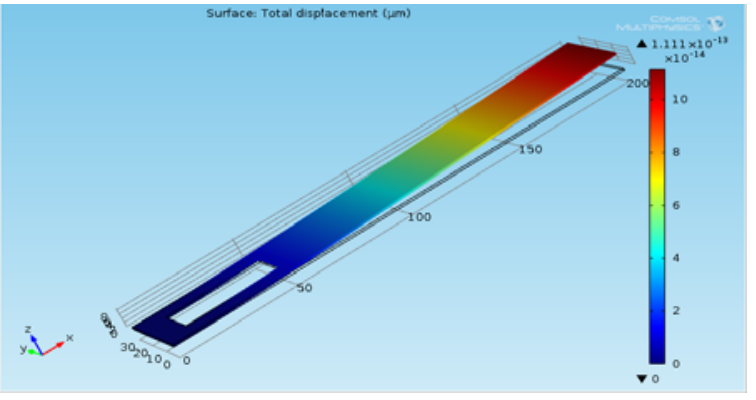

Figure 15. After reaction (both Antibody and Antigen)

\section{CONCLUSIONS}

The Micro-Cantilever designed exhibits both selectivity and sensitivity properties which play a role in Detection of N1H1 virus. This can be used to sense the Swine flu virus effectively. Also SiO2 was showing the high Sensitivity than other materials. We even changed the dimensions to alter the through-put of sensor.

\section{REFERENCES}

[1] M. Chaudhary, "Microcantilever-based Sensors," Defence Science Journal, vol/issue: 59(6), 2009.

[2] W. Zhou, "Simulation and design of piezoelectric microcantilever chemical sensors," Sciencedirect.

[3] S. Logeshkumar, "FEM based Estimation of Biological Interaction Using a Cantilever Array Sensor," Comsol conference Bangalore.

[4] R. Raiteri, "Micromechanical cantilever-based biosensors," Sensors and Actuators B 4010, pp. 1-12, 2001.

[5] N. K. Madzhi, "Design Simulation and Analysis of Polysiliconbased CMOS Micromachined Piezoresistive Microcantilever for Glucose Sensing," London, U.K., July 4 - 6, 2012.

[6] N. Nugaeva, "Micromechanical cantilever array sensors for selective fungal immobilization and fast growth detection," Biosensors and Bioelectronics, vol. 21, pp. 849-856, 2005.

[7] K. K. Zadeh and B. Fry, "Nano Technology-Enabled sensors," Springer.

[8] V. K. Varadan, et al., "NanoTechnology Engineering in nano and bio medicine".

[9] D. K. Parsediya, "Simulation and analysis of highly sensitive MEMS cantilever designs for "in vivo label free," biosensing, Procedia Technology, vol. 14, pp. 85 - 92, 2014.

[10] F. M. Battison, "A chemical sensor based on microfabricated cantilever array with simultaneous resonancefrequency and bending readout," sensors and actuators7 BB $145562644<6 \mathrm{C} 6$.

[11] R. A. Rahim, "Design and Analysis of MEMS Piezoresistive SiO2 Cantilever-based Sensor with Stress Concentration Region for Biosensing Applications," ICSE 2008 Proc., Johor Bahru, Malaysia, 2008.

[12] Rudresha K. J., "Microcantilever Optimization For MEMS Biosensor Applications," International Journal of Electronics \& Communication Engineering Research (IJECER), vol/issue: 1(1), 2013.

[13] A. Oliver, "Ambiguity Aversion and the UK Government's Response to Swine Flu," London School of Economics.

[14] S. Arora, "Design Of Mems Based Microcantilever Using Comsol Multiphysics," International Journal of Applied Engineering Research.

[15] P. G. Gopinath, "MEMS Microcantilevers Sensor Modes of Operation and Transduction Principles," International Journal of Computer Engineering Science (IJCES), vol/issue: 4(2), 2014.

[16] P. Ananthanarayan, "Microbiology," $8^{\text {th }}$ edition, universities press. 